\title{
CLOSED INTRAMEDULLARY RODDING FOR OSTEOGENESIS IMPERFECTA
}

\author{
R. W. D. MIDDLETON
}

From the Royal Alexandra Hospital for Children, Sydney

\begin{abstract}
Three cases of severe osteogenesis imperfecta are reported. Each was treated by closed intramedullary rodding, combined with osteoclasis to correct deformity. Operation was performed within a few months of birth. Both tibiae and both femora were stabilised in one operation, using $x$-ray image intensification to monitor placement of the rods. The technique used to insert the rods is described. The procedure appeared to be entirely satisfactory in reducing the incidence of fractures and it allowed the affected infants to be handled much more easily.
\end{abstract}

Correction of deformity combined with bone stabilisation has been used for many years in the treatment of osteogenesis imperfecta, notably since Sofield and Millar (1959) reported their technique of bone fragmentation and subsequent fixation with solid small-diameter rods. There do not appear to be any reports in the literature describing a closed method of inserting the rods, nor of this procedure being carried out within a few months of birth.

Three cases are reported showing the feasibility and effect of closed intramedullary rodding of the tibiae and femora at an early age in children with severe osteogenesis imperfecta.

\section{METHOD}

Under general anaesthesia, solid Sofield rods $3.2 \mathrm{~mm}$ in diameter were inserted by percutaneous puncture. $X$-ray image intensification was used to monitor the alignment and to ensure that rods of the correct length were used. Both tibiae and both femora were fixed in this way during the course of a single operation; tibial rods were inserted via the heel and femoral rods via the knee, no account being taken of the patella. In one case an attempt was made to insert rods into the humerus but great difficulty was experienced in visualising the bone.

A diamond-pointed Steinmann pin mounted in a power drill was used to make the initial track. Care was taken to select the appropriate entry point, as only angulation was possible once entry had been made. It was difficult to see the skeleton clearly on the screen of the image intensifier, since the ratio of bone density to soft-tissue density was poor. The rods were inserted with

R. W. D. Middleton, FRCS, FRACS, Orthopaedic Surgeon Department of Orthopaedic Surgery, Royal Alexandra Hospital for Children, Camperdown, New South Wales 2050, Australia.

(C) 1984 British Editorial Society of Bone and Joint Surgery

$0301-620 X / 84 / 5147 \$ 2.00$ a power drill, their length having been estimated from the image intensifier screen beforehand. It was necessary to ensure that the screw joining the drill to the rod was not so tight as to prevent disengagement of the rods after their placement.

In each case the deformity was corrected by osteoclasis at the tip of the rod as it was advanced along the intramedullary canal. No difficulty was experienced in correcting the alignment in these very young children. A similar attempt made in a child aged three years (not included in the present series) resulted in fractures occurring at unpredictable sites and not necessarily at the optimal position for correction of the deformity.

\section{CASE REPORTS}

Case 1. This child was the product of a full-term normal delivery. He was seen by his local doctor at two weeks of age because of a swollen left thigh. Radiographs revealed a recent fracture of the left femur and an old fracture of the right femur. Osteogenesis imperfecta was diagnosed. There was no family history of this disease and the only other sibling was normal. The child was admitted to hospital where he was treated by gallows traction. Whilst on traction a fracture of the right tibia occurred and subsequently he sustained a further fracture of his right femur (Figs 1 and 2).

At eight weeks of age percutaneous intramedullary rodding of the femora and tibiae was performed during the course of a single operation; at the same time the existing deformity was corrected by osteoclasis (Fig. 3). The extreme translucency of the bones made identification of their outlines difficult and the upper end of the right femur was incorrectly aligned. No splintage was used after the operation. The infant was pyrexial for two days but there was no clinical evidence of infection.

Subsequently he appeared to be much more content than before the operation and he was allowed home seven 


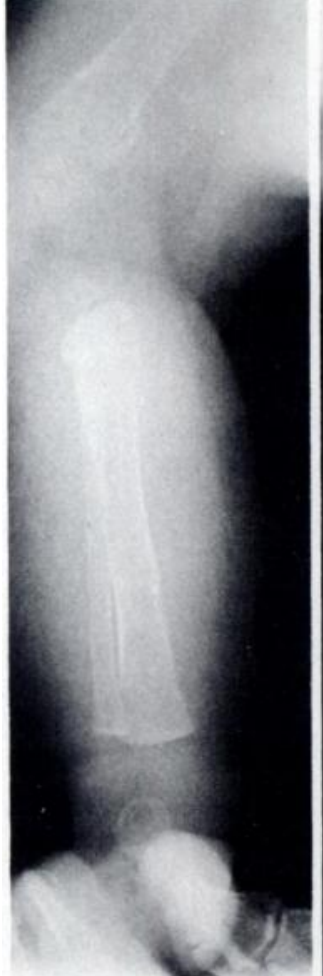

Fig. 1

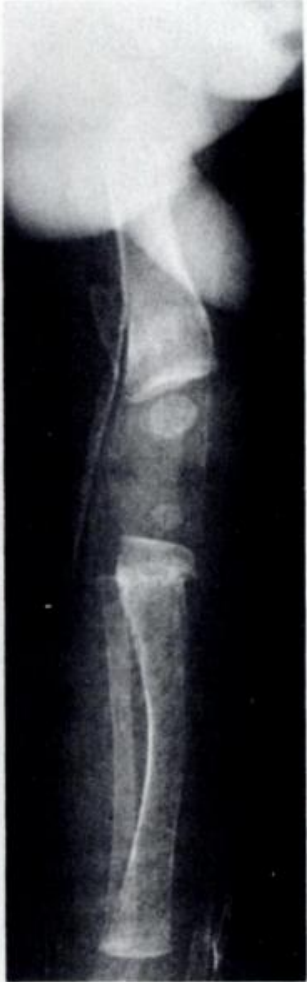

Fig. 2
Case 1. The lower limbs at the age of six weeks, with fractures of the femora and tibiae.

days after. Since that time he has had numerous metaphysial fractures (his mother thinks the number is about 40 ). The right femur has had fragmentation and rodding carried out once more, and the left femoral rod has been replaced. The original tibial rods remain in situ. Now, almost four years after the original operation, the boy is very active and his limbs are relatively undeformed (Figs 4 and 5).

Case 2. This child, with very severe osteogenesis imperfecta, was referred for treatment at 18 weeks of age because management of the skeletal fragility presented serious problems and his survival was threatened.

The child was very irritable and there was marked tibial bowing and fractures of the long bones (Fig. 6). There was no family history of osteogenesis imperfecta. Closed percutaneous rodding of the femora and tibiae with osteoclasis to correct deformity was performed at the age of 18 weeks (Fig. 7). After this the child became less irritable. At 32 weeks closed humeral rodding was carried out, but this was not as successful as the lower limb procedures.

Subsequently he has sustained fractures of the forearm bones, his right tibial rod has had to be replaced and he has fractured the right femur over the rod. Some three years after the initial rodding there is still some tibial bowing, but the femora remain fairly straight, and the bones are outgrowing the rods (Fig. 8). At the age of three-and-a-half years the child, who is cared for in an institution, is reported as being bright and happy.

Case 3. This child was admitted with fractures of the tibiae, femora and humerus, all of which had occurred since the age of two weeks. There was marked bowing of the femora and tibiae (Fig. 9). At 14 weeks of age, closed

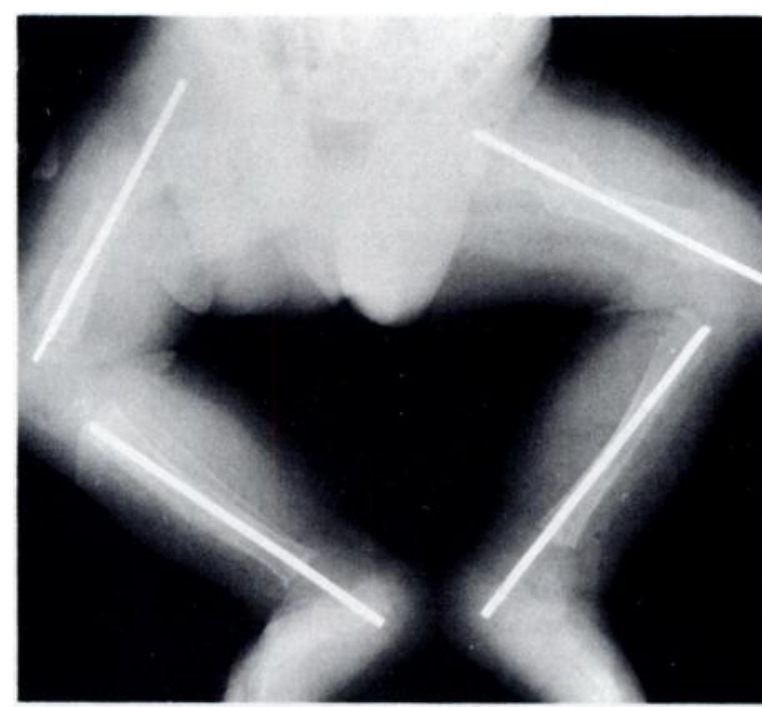

Fig. 3

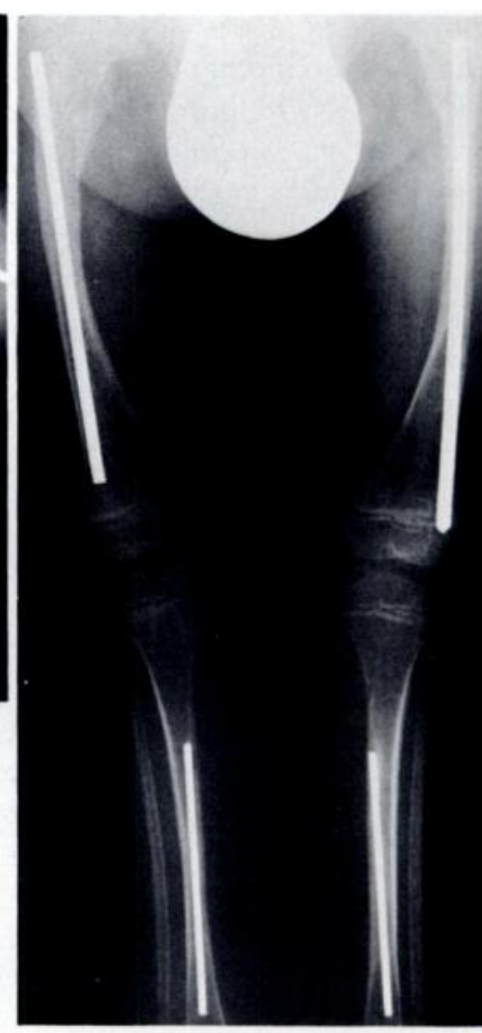

Fig. 4

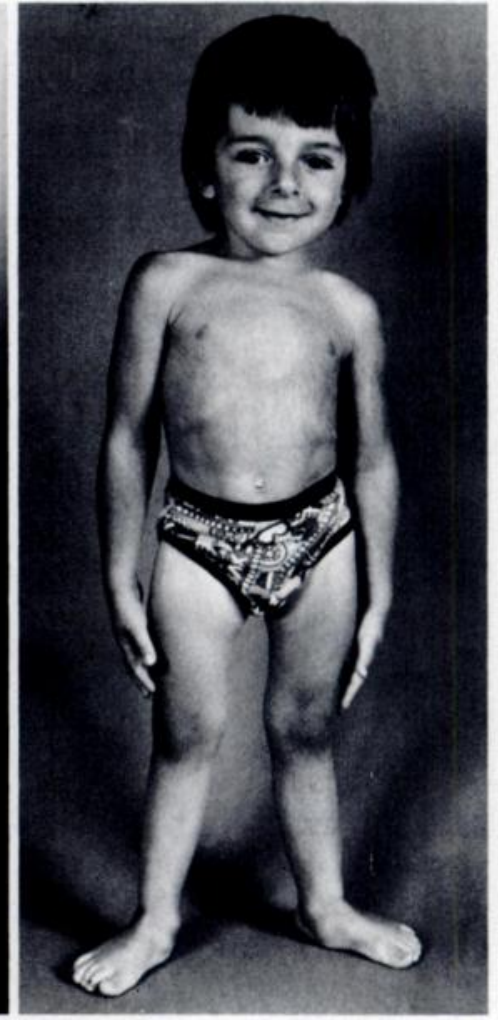

Fig. 5 


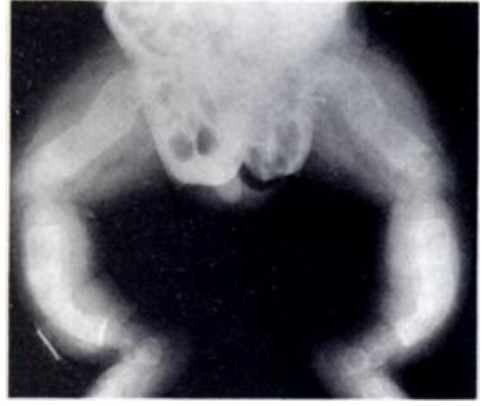

Fig. 6

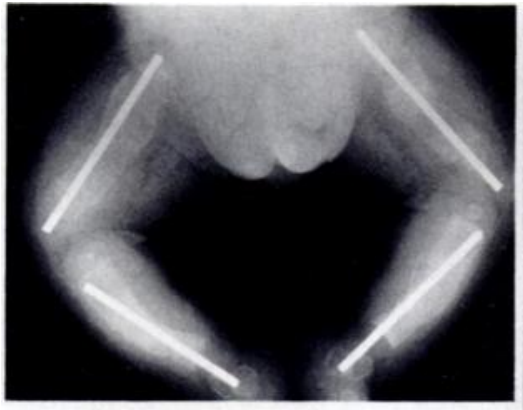

Fig. 7

Case 2. Figure 6-Both lower limbs when the child was 18 weeks old. Figure 7Appearance of the rods shortly after insertion. Figure 8-Three years after insertion; the original right tibial rod has been replaced.

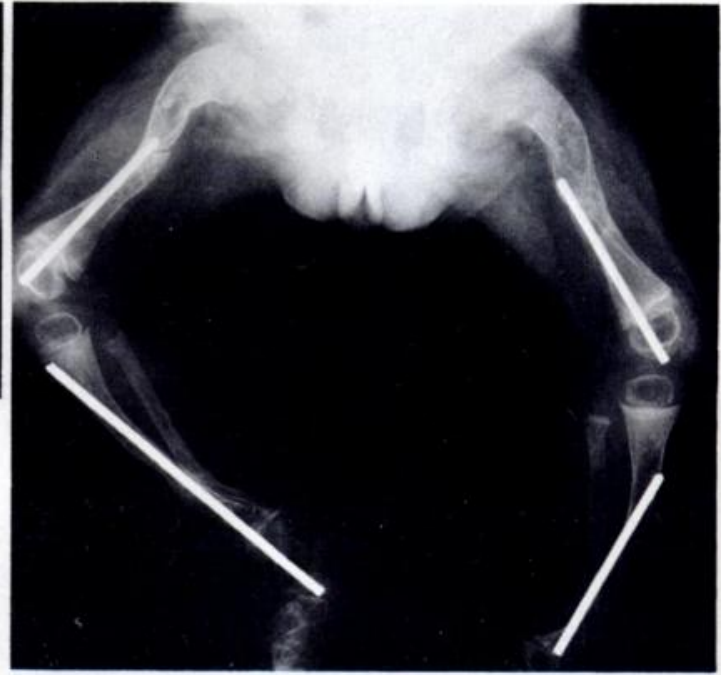

Fig. 8 rodding of the femora and tibiae combined with osteoclasis was performed at the one operation (Fig. 10). After operation the limbs were swollen and the child had a temperature of $38^{\circ} \mathrm{C}$. No blood transfusion was given and the haemoglobin stabilised at $7.7 \mathrm{~g} / \mathrm{dl}$. He was allowed home seven days after operation. Subsequently, his parents reported that he was a much happier child. Now, at the age of 13 months he has had no further lower limb fractures (Fig. 11).

\section{DISCUSSION}

Intramedullary rodding of the long bones of children suffering from osteogenesis imperfecta reduces the frequency of fractures and prevents the development of severe secondary deformity. In association with corrective osteotomies at the time of rodding, the procedure described is of significant value in improving the lifestyle of the affected children. The improvement persists into adult life, so that the majority of these people retain their ability to walk and as adults they are generally productive and socially adaptable (Moorefield and Miller 1980).

Many of the most severe cases of the disease perish in the neonatal period. In those who survive, the ordinary handling needed for infant care presents great difficulty; even with the utmost care fractures are frequent. The children also tend to be apprehensive and irritable thus adding to the problems.

The three cases reported indicate that it is possible, without multiple open osteotomies, to correct deformity and to provide skeletal stability at a very early age. After operative treatment the children were reported to be less anxious and less irritable. Despite the severe nature of the disorder, in all cases the treated infants have thrived. Those who look after these children have all agreed that, after treatment, the children were easily handled and appeared to be happier than would otherwise have been expected; presumably this is because their limbs are more comfortable.

The problem of bone overgrowing the rods remains,

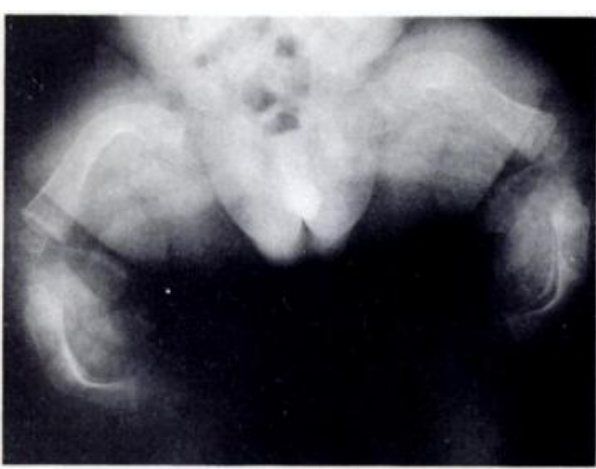

Fig. 9

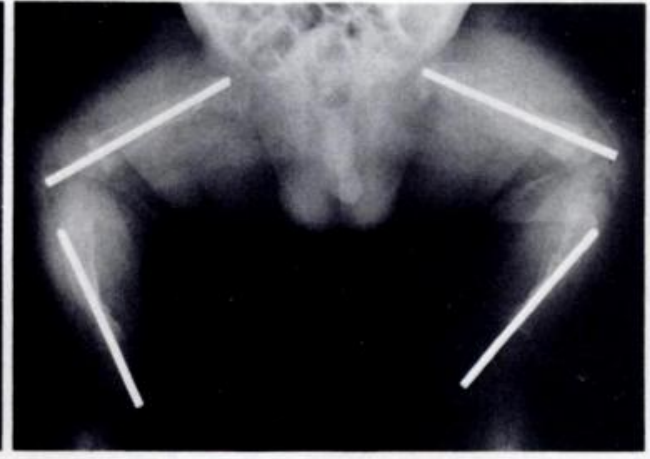

Fig. 10

Case 3. Figure 9-The lower limbs of the child at the age of 14 weeks. Figure 10-Appearance of the rods shortly after insertion. Figure 11 -Appearance 10 months after rodding.

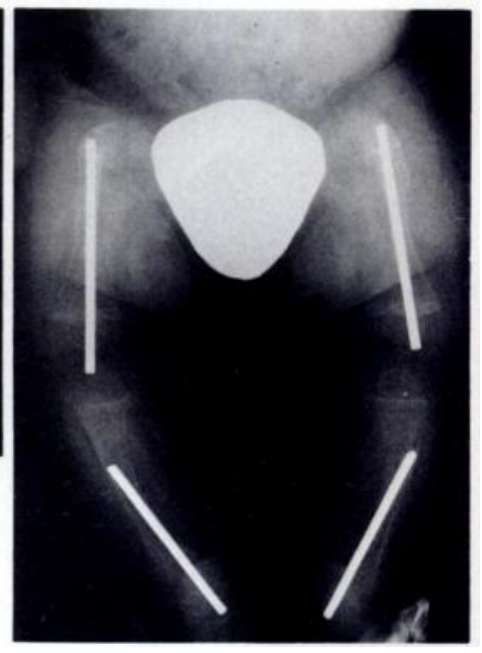

Fig. 11 
as it does when rodding is performed in older children. The closed nature of the procedure and the size of the medullary canal prevented the use of expandable rods. The rods were deliberately left overlong to try to increase their useful life, and no problems resulted from transfixing the ankle and subtalar joints; protrusion into the knee also caused no difficulty. In fact bone rapidly overgrew the rods. Persistent central breach of the growth plate has not apparently caused any complication, a fact which has been noted previously (Marafioti and Westin 1977).
However, some rods became completely incorporated in the diaphysis; what problems this may cause, especially with retrieval, remains to be seen.

Conclusion. In the cases reported, early closed rodding of the lower limb bones of children severely affected by osteogenesis imperfecta has improved the early management of the disorder without apparently causing any illeffects. Hospitalisation is short, major operative exposure of bone is avoided and, most important, four bones can be fixed at one operation.

\section{REFERENCES}

Marafioti RL, Westin GW. Elongating intramedullary rods in the treatment of osteogenesis imperfecta. J Bone Joint Surg [Am] 1977:59-A: 467-72.

Moorefield WG Jr, Miller GR. Aftermath of osteogenesis imperfecta: the disease in adulthood. J Bone Joint Surg [ Am] 1980;62-A: 11 3-9.

Sofield HA, Millar EA. Fragmentation, realignment and intramedullary rod fixation of deformities of the long bones in children: a ten-year appraisal. J Bone Joint Surg [Am] 1959;41-A: 1371-91. 